\title{
Correlation between age, muscle architecture, and muscle strength in children with Erb's palsy
}

\author{
Ahmed Saad Awad ${ }^{1,2^{*}}$, Mostafa Soliman Ali ${ }^{1}$ and Mohamed Ismail Elassal ${ }^{1}$
}

\begin{abstract}
Background: Muscle weakness is a widespread problem in children with Erb's palsy as it can cause changes in muscle architecture parameters, which can be detected by ultrasonography. This study was conducted to determine the relation between age, muscle architecture, and muscle strength in children with Erb's palsy. A total of 40 children with Erb's palsy from both sexes aged 1-2.5 years were included in this study. Muscle thickness and pennation angle were measured by ultrasonography, and muscle strength was measured using the active movement scale.
\end{abstract}

Results: A significant relation was found between age, muscle thickness, pennation angle, and muscle strength ( $P$ $<0.05)$. Moreover, a significant difference was found in muscle architecture parameters during relaxation and contraction in both study groups and in each study group $(P<0.05)$.

Conclusion: Muscle weakness in children with Erb's palsy has an effect on muscle architecture parameters, and these parameters also increase with age.

Keywords: Erb's palsy, Pennation angle, Muscle thickness, Muscle strength

\section{Key messages}

There is a strong correlation between muscle architecture and activity.

The active movement scale can be used to evaluate muscle strength in infants with Erb's palsy without the need of cooperation.

Muscle thickness and pennation angle increase markedly with muscle contraction.

\section{Background}

Brachial plexus palsy (BPP) is an injury to the total or a part of the brachial plexus during birth. The incidence of BPP has been reported to be $0.42-4.6$ per 1000 live births, with variable degrees of severity [1]. Erb's palsy includes affection of the C5 and C6 roots of the brachial plexus, which causes weakness of several muscles such

\footnotetext{
* Correspondence: elwahsh2004@gmail.com

${ }^{1}$ Cairo University, Giza, Egypt

${ }^{2}$ Giza, Egypt
}

as the deltoid, biceps, brachialis, infraspinatus, supraspinatus, and serratus anterior muscles; the rhomboids, levator scapulae, and supinator muscles are also involved [2]. The injured upper limb is held in a "Waiter's tip" appearance, which presents extension and internal rotation of the arm, pronation of the forearm, and flexion of the wrist. Absence of the Moro reflex in the arm with intact grasping of the hand in the same side indicates that the case is diagnosed as Erb's palsy [3].

Examination of the musculoskeletal system is one of the most important applications for diagnostic radiological imaging [4]. Contractility is a property of muscle tissue that enables it to adapt its architecture to different stresses. In addition, the structure of the muscle correlates strongly with its activity [5]. One of the primary determinants of muscle function is its architecture, which is the internal arrangement of fibers within the muscle in relation to the axis of force generation [6]. Muscle ultrasound is a convenient technique that can be used to

\section{Springer Open}

(c) The Author(s). 2021 Open Access This article is licensed under a Creative Commons Attribution 4.0 International License, which permits use, sharing, adaptation, distribution and reproduction in any medium or format, as long as you give appropriate credit to the original author(s) and the source, provide a link to the Creative Commons licence, and indicate if changes were made. The images or other third party material in this article are included in the article's Creative Commons licence, unless indicated otherwise in a credit line to the material. If material is not included in the article's Creative Commons licence and your intended use is not permitted by statutory regulation or exceeds the permitted use, you will need to obtain permission directly from the copyright holder. To view a copy of this licence, visit http://creativecommons.org/licenses/by/4.0/. 
visualize the normal and pathological muscle tissue as it is noninvasive and can be performed in real time [7]. Measurements of muscle architecture comprise muscle thickness quantification, pennation angle, and fascicle length in addition to physiological and anatomical crosssectional areas [8]. Muscle thickness is evaluated by measuring the perpendicular distance between the superficial and deep aponeuroses on the image [6]. Various studies have shown that muscle thickness can be an indirect indicator of muscle strength [9]. A direct relationship exists between the number of sarcomeres that are arranged in parallel (muscle size) and the amount of force that is produced by the muscle [8]. Pennation angle is evaluated by measuring the angle between the deep aponeurosis and the line of the fascicle [8]. The pennation angle could change up to $120-170 \%$ between rest and maximum isometric contractions at a definite joint angle. Therefore, neglecting this effect of contraction on the pennation angle can lead to large miscalculations of muscle force and joint moment [10]. The US quantification of the muscle architecture provides information about the effects of neuromuscular disorders on the muscle or the documentation of treatment effects [6].

The active movement scale (AMS) is a reliable tool to evaluate muscle strength in infants with obstetric BPP [11]. The AMS has several advantages as it can be suitably used in small children and does not require performing the task on demand. Moreover, the joint is assessed totally, not only on individual muscle testing, and the scale can be used in both preoperative and postoperative cases. In addition, the first 4 grades are scored according to the improvement throughout the entire range of motion with elimination of gravity, which already indicates a muscle contraction but without useful function. Consequently, the useful muscle contraction begins when the patient is midway to full recovery [12].

Understanding the muscle architecture parameters and their changes according to age and muscle activity can help in designing the rehabilitation program and to select the suitable exercises that can affect muscle strength and thereby its architecture. We hypothesized that a relation exists between age, muscle architecture, and muscle strength in children with Erb's palsy.

\section{Methods}

This study was conducted at the outpatient clinic of the Faculty of Physical Therapy, Cairo University, in 2019 according to the code of Ethics of the World Medical Association (Declaration of Helsinki) for experiments involving humans. Ethics approval from the Faculty of Physical Therapy, Cairo University, Egypt (no. P.T.REC/ 012/002232), and a signed written consent form with parent acceptance for participation in the study and publication of the results were obtained before starting the study procedures. A total of 40 children with Erb's palsy selected from both sexes aged from 1 to 2.5 years were included in this study. The inclusion criteria were children with Erb's palsy with lesion only at the C5 and C6 nerve roots, having persistent muscle weakness, and with adequate cooperation to allow the radiologist to position the arm in the correct manner for ultrasonography. The exclusion criteria were children with lesion at other roots of the brachial plexus and with a previous history of upper limb trauma or surgery in the affected and nonaffected sides.

Sample size was calculated according to the assumption of presence of significant correlation between muscle thickness and age in either affected or nonaffected sides, with $\alpha=0.05$, two sided, power of $80 \%$, and correlation coefficient equal to 0.43 . So a sample size of 40 patients would be required (GPower 301 http://www. psycho.uni-duesseldorf.de)

Ultrasonography (device type GE LOGIQ P6) with a frequency of $7.5 \mathrm{MHz}$ was used to measure muscle thicknesses and pennation angles of the anterior deltoid muscle [8]. In addition, the AMS was used to measure the strength of the anterior deltoid muscle [11].

First, the researcher collected the personal details from the children's parents, including name, age, and address. Then, the protocol and the benefits of the study for their children were explained to the parents.

Muscle architecture parameters (pennation angles and muscle thicknesses) of the anterior deltoid muscle were measured by ultrasonography during contraction and relaxation. First, the child was placed comfortably in the supine or sitting position facing the radiologist with the shoulder in the anatomical position. To obtain accurate measurement, the radiologist used a suitable amount of contact gel for acoustic coupling and performed the procedure without exerting high pressure on the muscle. A longitudinal section image was taken at the middle of the anterior deltoid muscle, while the researcher was seated beside the patient to guide the child's arm during relaxation and contraction. If the child did not understand the order of contraction by demonstrating the shoulder flexion, the researcher stimulated the child to contract the anterior deltoid muscle by scratching or squeezing over the muscle. After capturing the images by ultrasonography, the images were entered into the personal computer of the researcher in the "AutoCAD" program that was used to measure the tested parameters. The pennation angle was measured by drawing two lines; the first line was drawn parallel to the fascicle, and the second line was drawn parallel to the deep aponeurosis or bone. The angle between the two lines represented the pennation angle. Muscle thickness was measured by drawing a vertical line between the superficial aponeurosis and the deep aponeurosis or bone. 
Measurements were taken three times for each parameter (in each image), and the mean of the three measurements was calculated, recorded, and saved to an Excel sheet. These steps were performed for images of both contraction and relaxation as well (Figs. 1 and 2).

For measurement of muscle strength, the examiner used the AMS to check the anterior deltoid muscle. This scale assesses the muscle strength with elimination of gravity and against gravity. First, the researcher started testing with elimination of gravity (such as the side-lying position). The child was placed in the side-lying position with shoulder adduction and elbow extension and was encouraged to flex his/her shoulder by squeezing the anterior deltoid muscle or by trying to touch a toy. Then, the child was given a grade for the anterior deltoid muscle according to the AMS. The nonaffected side was tested in the same manner and was used as a reference for comparisons. If the child completed the range with elimination of gravity, the researcher started the testing against gravity from an upright position such as sitting or standing with the upper limb beside the trunk. The researcher encouraged the child to flex his/her shoulder against gravity and gave the child a grade according to the AMS (Fig. 3).

\section{Data analysis}

Results are expressed as mean \pm standard deviation. For assessing normality, the Kolmogorov-Smirnov test was used to measure the distribution of data. Accordingly, for data that were normally distributed, the comparison between variables in the two sides was performed using an unpaired $t$-test. Comparison between relaxation and contraction status was performed using a paired $t$-test. The correlation between different variables was evaluated using the Pearson correlation coefficient. The Statistical Package for Social Sciences computer program (version 19 windows) was used for data analysis. $P$ value $\leq 0.05$ was considered to be statistically significant.

\section{Results}

The age of the study participants ranged from 1.0 to 2.5 years, with a mean $( \pm \mathrm{SD})$ age of $1.65 \pm 0.55$ years. The sample included 23 boys (57.5\%) and 17 girls (42.5\%) (Table 1).

There was a significant difference in muscle thickness and pennation angle during contraction and relaxation between the two examined sides, except for the pennation angle during relaxation that showed no significant difference. In addition, a significant difference was found in muscle thickness and pennation angle during contraction and relaxation in each study group (Table 2).

Furthermore, there was a significant correlation between age, muscle thickness, pennation angle, and muscle strength (AMS), but there was no relationship between muscle thickness and muscle strength (Figs. 4, 5, 6, 7, and 8).

\section{Discussion}

This study was conducted to analyze the relation between age, muscle architecture, and muscle strength in children with Erb's palsy and to compare the architecture parameters in both study groups during contraction and relaxation and also in each group between

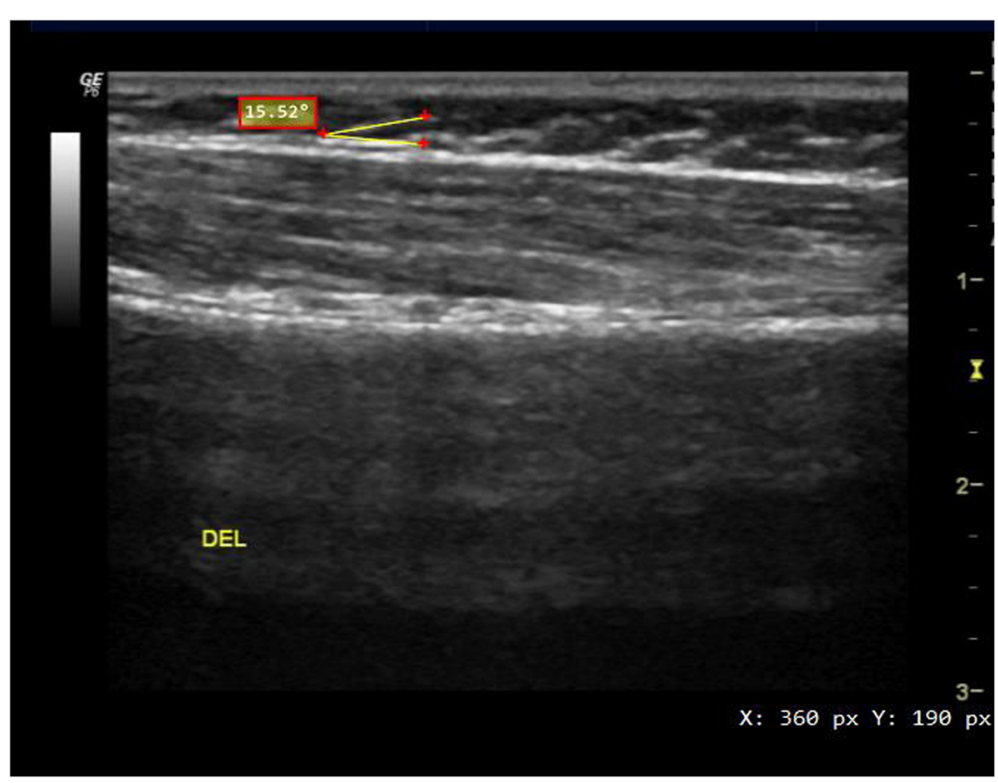

Fig. 1 Measurement of pennation angle of the anterior deltoid muscle by ultrasonography 


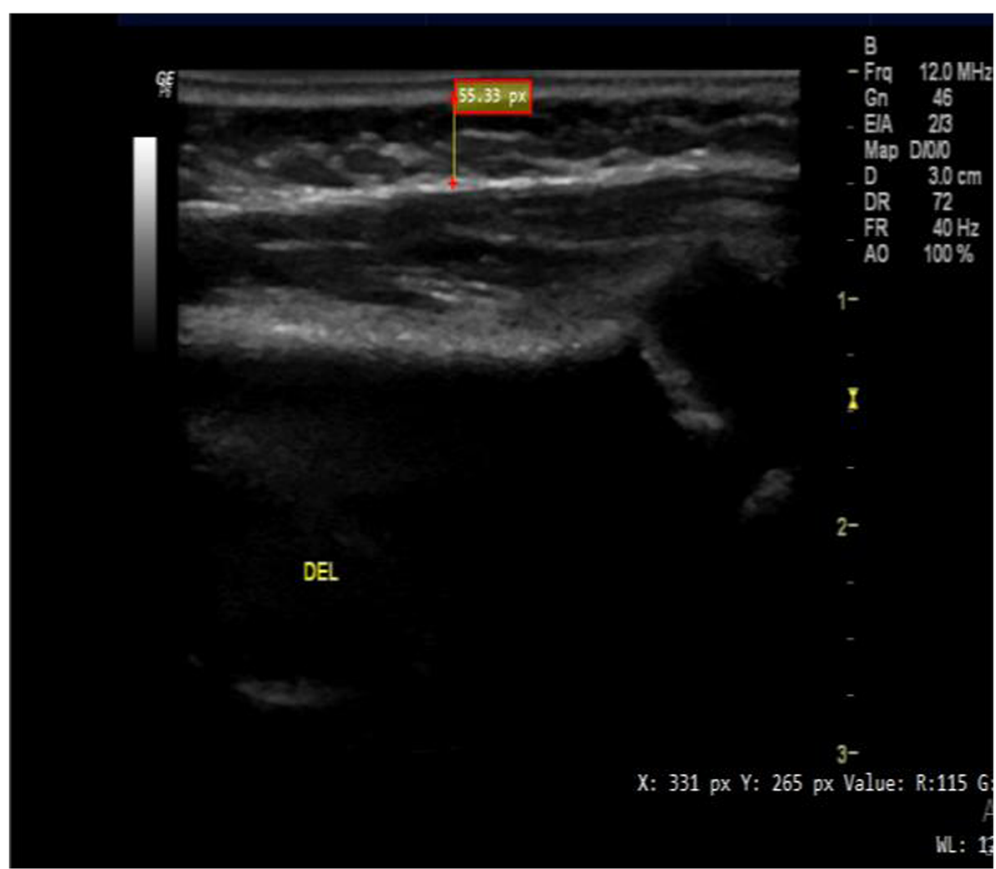

Fig. 2 Measurement of muscle thickness of the anterior deltoid muscle by ultrasonography

relaxation and contraction. As the majority of children with Erb's palsy have abnormal reaching as they reach with an abducted arm, this refers to the weakness of the anterior deltoid muscle that is responsible for shoulder flexion. Our results confirmed the presence of a significant correlation between age, muscle thickness, pennation angle, and muscle strength, but no relationship was detected between muscle thickness and muscle strength in the affected side. Furthermore, a significant difference was found in muscle architecture parameters during contraction and relaxation between the two examined

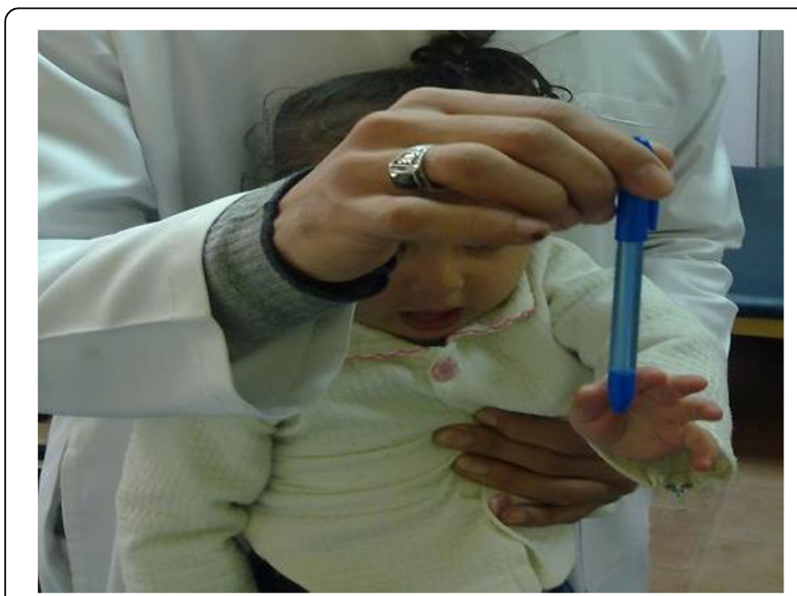

Fig. 3 Application of the active movement scale for the anterior deltoid muscle sides and also between contraction and relaxation in each side. The significant correlation between age and muscle architecture parameters found in this study is consistent with a previous report [13] that showed that pennation angle values increase from birth and stabilize after adolescence. Our results are also consistent with another study [14] wherein it was found that the muscle thickness increases with age from 2 to 16 years in healthy children. The increase in the muscle architecture parameters with age may be due to the ongoing maturation and development of children as the muscle size increases.

The significant correlation between muscle thickness and pennation angle found in the present study is also reinforced by a previous investigation [15], which analyzed the relationship between pennation angle and muscle thickness in normal individuals and observed a significant correlation. In addition, another previous

Table 1 General characteristics of the study subjects

\begin{tabular}{ll}
\hline Mean age $( \pm$ SD) & $\mathbf{1 . 6 5} \pm \mathbf{0 . 5 5}$ \\
\hline Affected side & $21(52.5 \%)$ \\
Right & $19(47.5 \%)$ \\
Left & \\
Sex & $23(57.5 \%)$ \\
$\quad$ Boys & $17(42.5 \%)$ \\
Girls &
\end{tabular}


Table 2 Comparison between the mean values of muscle thickness and pennation angle (relaxation and contraction) in the two examined sides and in each side

\begin{tabular}{|c|c|c|c|}
\hline & Nonaffected $(n=40)($ mean \pm SD) & Affected $(n=40)($ mean \pm SD) & $P$ value \\
\hline \multicolumn{4}{|c|}{ Muscle thickness (cm) } \\
\hline Relaxation & $1.15 \pm 0.23$ & $1.05 \pm 0.23$ & $0.043(S)$ \\
\hline Contraction & $1.29 \pm 0.24$ & $1.14 \pm 0.23$ & $0.006(S)$ \\
\hline$P$ value & $0.001(S)$ & $0.001(S)$ & \\
\hline \multicolumn{4}{|c|}{ Pennation angle (degrees) } \\
\hline Relaxation & $13.55 \pm 2.21$ & $12.72 \pm 2.03$ & 0.085 (NS) \\
\hline Contraction & $15.12 \pm 2.24$ & $13.62 \pm 2.23$ & $0.004(S)$ \\
\hline$P$ value & $0.001(S)$ & $0.001(S)$ & \\
\hline
\end{tabular}

NS, $P>0.05=$ not significant; $S . P<0.05=$ significant

study [13] showed that the pennation angle has a direct relationship with muscle thickness. Furthermore, a correlation between pennation angle and muscle volume has been reported [16]. The results of the present study revealed a significant correlation between pennation angle and muscle strength, which is consistent with the previous study [16] reporting an increase in the pennation angle after strength training of the vastus lateralis muscle that increases the muscle volume by $10 \%$. However, a negative correlation between pennation angle and joint force has been reported [17]. As the muscle volume increases, the muscle thickness and the pennation angle increase as a normal response to increasing age or to strength training.

The nonsignificant correlation found between muscle thickness and strength in this study is in contrast to a previous report [18], which showed that muscle strength is related to muscle volume as indicated by the muscle cross-sectional area and thickness. In addition to these results, another study [19] found that there is a strong relationship between the thickness of the quadriceps muscle and maximum voluntary contraction. According to these results, a nonsignificant correlation exists between muscle thickness and strength of the anterior deltoid muscle in children with Erb's palsy. However, we observed that muscle thickness was significantly reduced in the affected side. This could be due to the little difference at young age (from 1 to 5 years), but the correlation between muscle thickness and strength may change over time during the growth period due to lower mobility, disuse atrophy, and muscle weakness. Furthermore, these differences were associated with changes in muscle strength; therefore, an appropriate exercise program designed to improve muscle strength during the

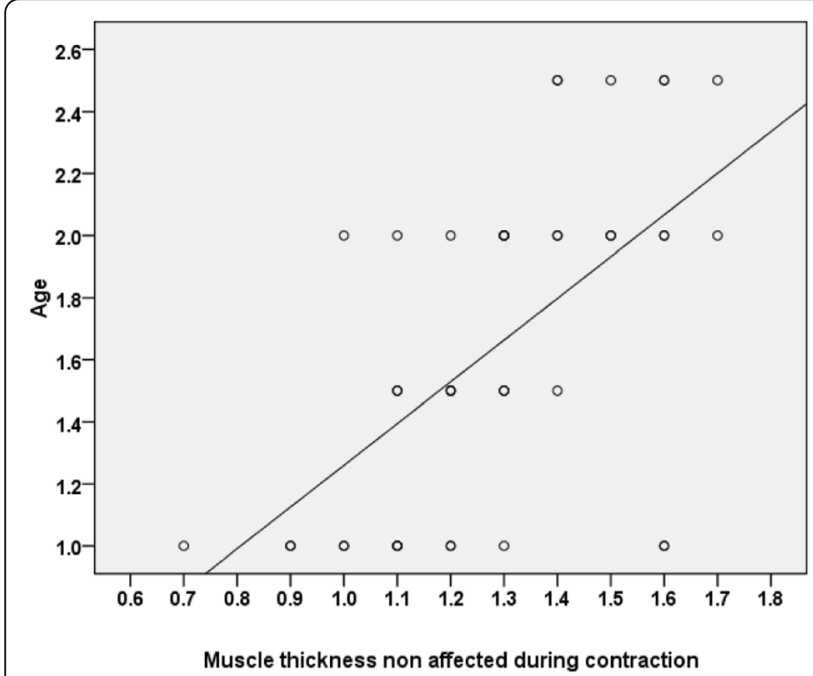

(a)

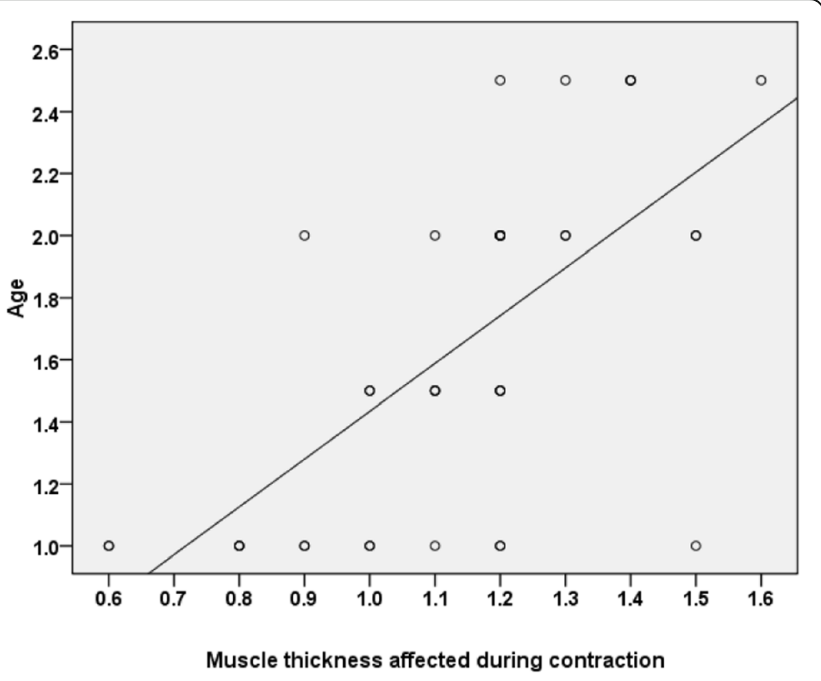

(b)

Fig. 4 a Correlation between age and muscle thickness in the nonaffected side during contraction $(r=0.596 ; P=0.001)$. b Correlation between age and muscle thickness in the affected side during contraction $(r=0.658 ; P=0.001)$ 


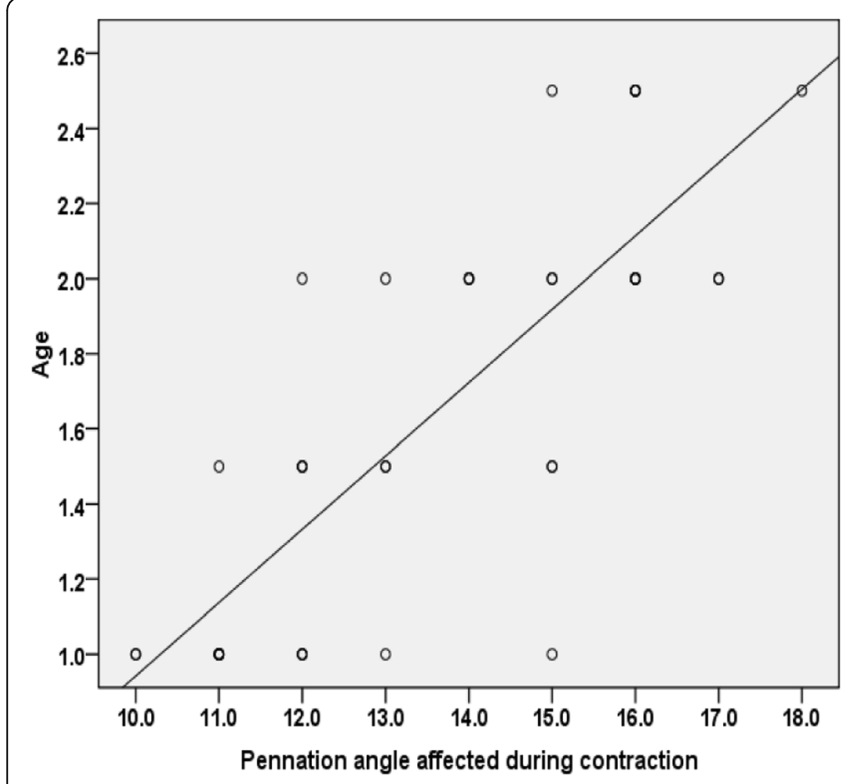

(a)

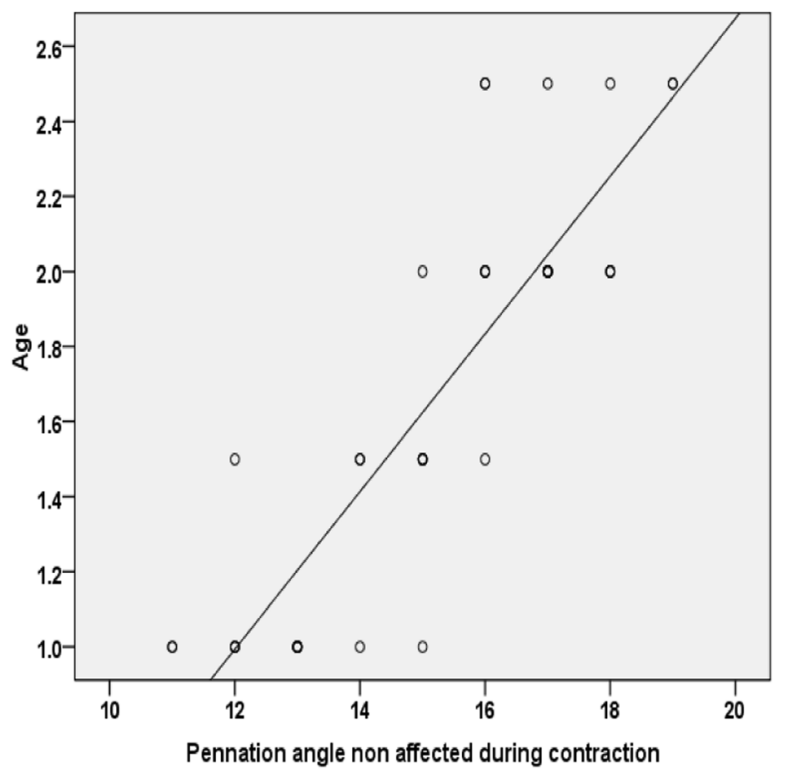

(b)

Fig. 5 a Correlation between age and pennation angle in the affected side during contraction $(r=0.797 ; P=0.001)$. b Correlation between age and pennation angle in the nonaffected side during contraction $(r=0.864 ; P=0.001)$

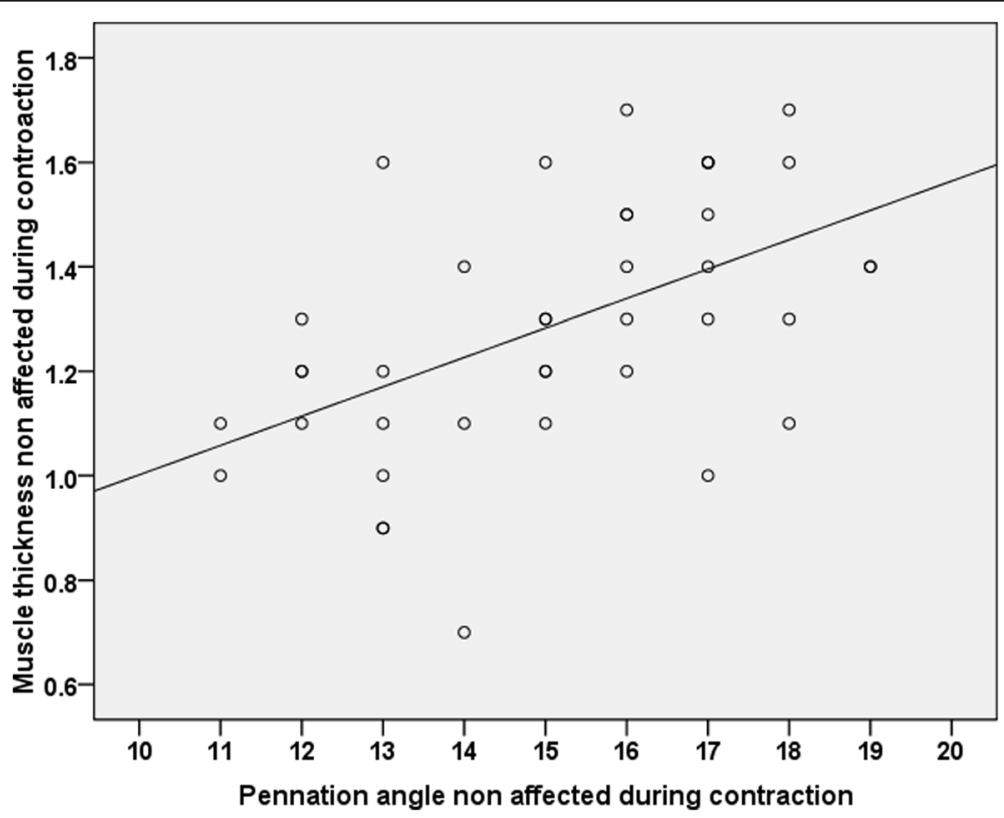

Fig. 6 Correlation between muscle thickness in the nonaffected side during contraction and pennation angle in the nonaffected side during contraction $(r=0.523 ; P=0.001)$ 


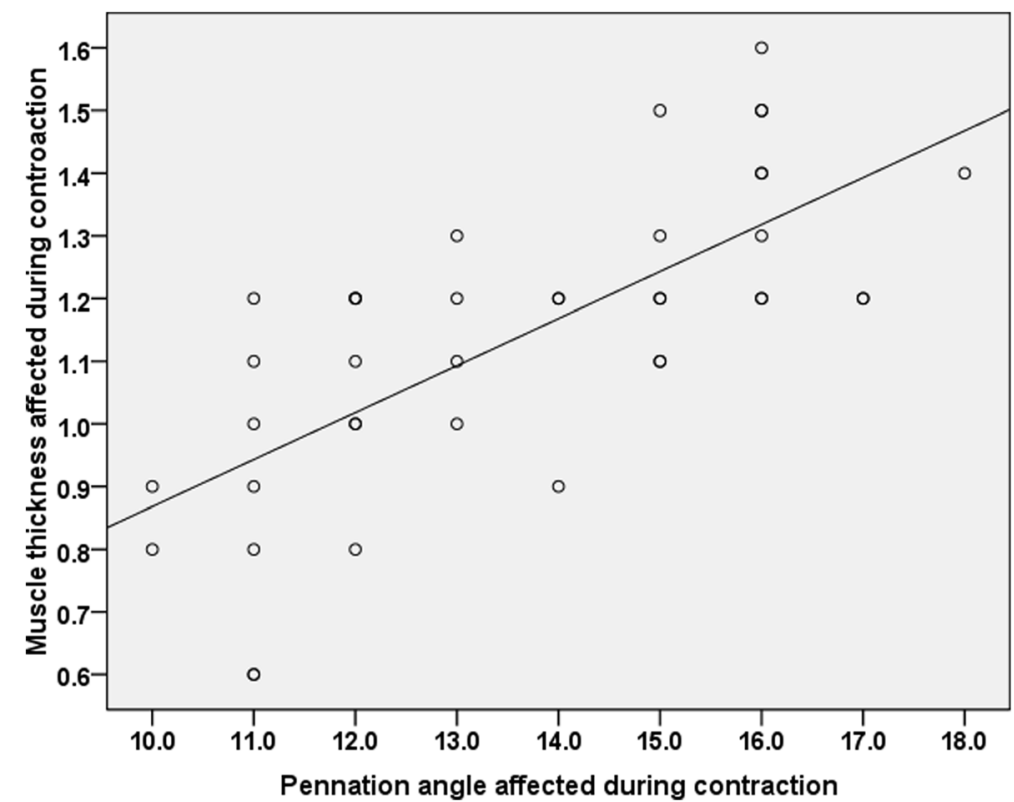

Fig. 7 Correlation between muscle thickness in the affected side during contraction and pennation angle in the affected side during contraction $(r=0.717 ; P=0.001)$

growth spurt may be required. Therefore, the results of this study could not reveal the time required for muscle thickness to change with age, which indicates that a large sample size is required to generalize this result and make further predictions and assumptions to investigate the relationship of muscle thickness and muscle strength with age in children [20]. Also, this nonsignificant correlation may be due to the lack of activity of these children at this young age, which is exacerbated by the neurological affection that limits the muscle thickness and strength to some extent.

The results of this study also showed that there is a significant difference in muscle architecture parameters during relaxation and contraction between the two examined sides, except for the pennation angle during relaxation that showed no significant difference. The significant difference between both sides in the pennation angles during contraction is consistent with a

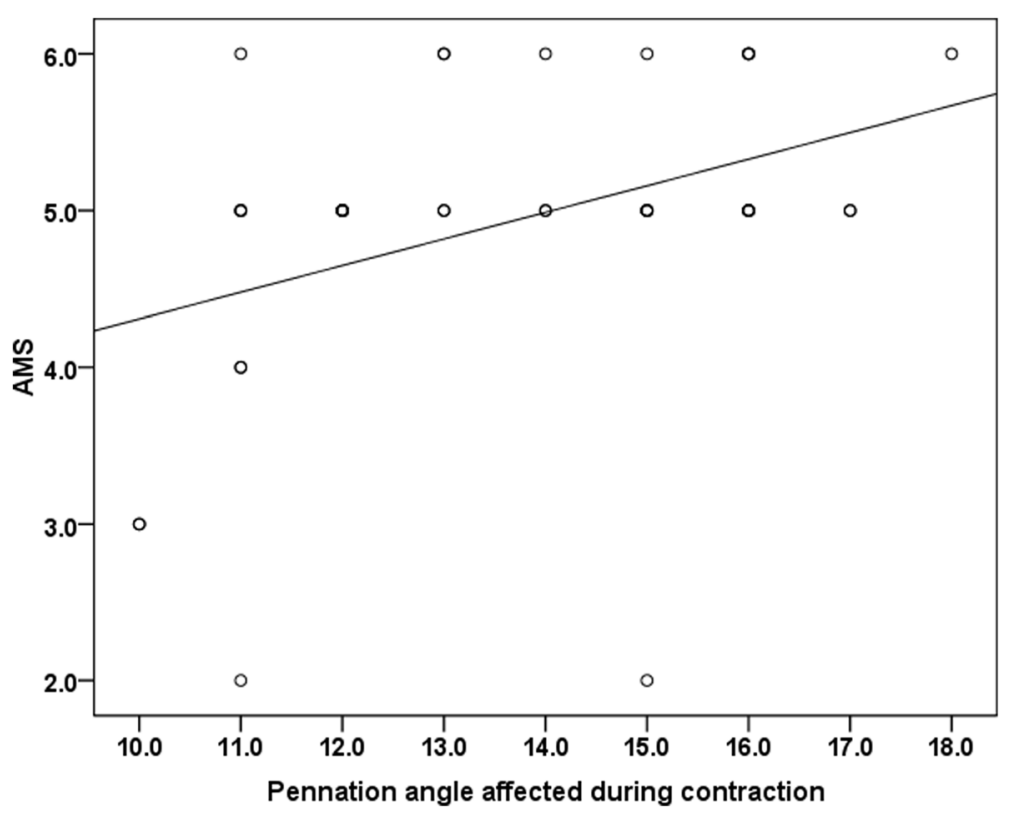

Fig. 8 Correlation between AMS and pennation angle in the affected side during contraction $(r=0.390 ; P=0.013)$ 
previous report [21] wherein the authors claimed that in case of disuse atrophy, the pennation angle of muscle fibers is reduced compared to that in healthy young persons, which is largely due to the decreased amount of contractile tissue. However, this finding does not agree with the results of comparison of pennation angles during relaxation. It has been reported that a decreased muscle size is associated with changes in fascicle length and pennation angle after a time of disuse [22]. On the other hand, in a previous study [23], the authors claimed that with a decrease in physical activity, there were no significant changes in muscle architecture in the antigravity muscles such as the biceps brachii and tibialis anterior; however, this agrees with our results only in the comparison of pennation angles during relaxation between both groups. This significant difference in muscle architecture between both sides may be due to the muscle weakness and the decrease of functional activities in the affected side. Therefore, we expected a marked difference in the pennation angle during relaxation that exhibited a nonsignificant difference.

In addition, the significant increase in the muscle architecture from relaxation to contraction in each side is in agreement with an earlier report [24] showing that the pennation angle of a given muscle increases as the muscle fibers shorten, that is, muscle fiber rotation occurs during fiber shortening. Another study [25] found that the thickness of the proximal semitendinosus muscle increased with contraction on both the unaffected and affected sides after hamstring strain injury. The significant increase in muscle thickness and pennation angle from relaxation to contraction observed in the present study may be due to the increase in muscle size during contraction that alters the muscle architecture.

\section{Limitations of the study}

The current study has some limitations. First, measurements were performed only on the middle area of the muscle; it has been shown that the muscle architecture can vary throughout the muscle length. Secondly, the study was limited to measurement of muscle thickness and pennation angle, whereas other parameters such as fascicle length were not measured. Thirdly, the study was limited to evaluation of only the anterior deltoid muscle. Fourthly, the sample size of participant population is small.

\section{Conclusion}

Muscle weakness in children with Erb's palsy may affect muscle thickness and pennation angle at this age, and as age advances, these parameters will change. Therefore, while designing a rehabilitation program for these children in this age group, it is important to consider the changes in the muscle architecture of the affected side to achieve the appropriate function.

\section{Abbreviations}

BPP: Brachial plexus palsy; AMS: Active movement scale; US: Ultrasonography; SD: Standard deviation; S: Significant; NS: Not significant

\section{Acknowledgements}

We appreciate the role of parents of children who participated in this study. Also, the great effort of the Egyptian Knowledge Bank to edit this manuscript.

\section{Authors' contributions}

The authors testify that all persons designated as authors qualify for authorship. ASA was involved in the study concept and design, interpretation of data, and writing of the initial and final drafts of the article. MSA and MIE were involved in data acquisition and analysis. All authors have critically reviewed and approved the final draft and are responsible for the content and similarity index of the manuscript.

\section{Funding}

Nil

\section{Availability of data and materials}

The data collected and/or analyzed during the study are available from the corresponding author on reasonable request and after institutional approval.

\section{Declarations}

Ethics approval and consent to participate

Ethics approval from the Faculty of Physical Therapy, Cairo University, Egypt (no. P.T.REC/012/002232), and a signed written consent form with parent acceptance for participation in the study and publication of the results were obtained before starting the study procedures.

Consent for publication

Not applicable

\section{Competing interests}

The authors declare that they have no competing interests.

Received: 26 December 2020 Accepted: 20 April 2021

Published online: 09 June 2021

\section{References}

1. Evans-Jones G, Kay SPJ, Weindling AM, Cranny G, Ward A, Bradshaw A, et al. Congenital brachial palsy: incidence, causes, and outcome in the United Kingdom and Republic of Ireland. Arch Dis Child Fetal Neonatal Ed. 2003;88: 185-9.

2. Greenfield BH, Geist K. Evaluation and treatment of brachial plexus lesions. In: Donatelli RA, ed. Physical Therapy of the Shoulder, 5th ed. Saint Louis: Churchill Livingstone; 2011. p. 163-83.

3. Alexander, Mathews DJ. Pediatric rehabilitation: principles and practice. 4th ed: Demos Medical Publishing; 2010.

4. Reginelli A, Zappia M, Barile A, Brunese L. Strategies of imaging after orthopedic surgery. Musculoskelet Surg. 2017;101(S1):101.

5. Debernard L, Robert $\mathrm{L}$, Charleux F, Bensamoun SF. Characterization of muscle architecture in children and adults using magnetic resonance elastography and ultrasound techniques. J Biomech. 2011;44:397-401.

6. Legerlotz K, Smith HK, Hinge WA. Variation and reliability of ultrasonographic quantification of the architecture of the medial gastrocnemius muscle in young children. J Clin Physiol Function Imaging. 2010;30:198-205.

7. Pillen S. Skeletal muscle ultrasound. Eur J Transl Myol. 2010;1:145-55.

8. Moreau NG, Simpson KN, Teefey SA, Damiano DL. Muscle architecture predicts maximum strength and is related to activity levels in cerebral palsy. Phy Ther. 2010;90:1619-30.

9. Ohata K, Tsuboyama T, Haruta T, Ichihashi N, Nakamura T. Longitudinal change in muscle and fat thickness in children and adolescents with cerebral palsy. Dev Med Child Neurol. 2009:5:943-8.

10. Maganaris CN, Baltzopoulos $V$, Sargeant AJ. In vivo measurements of the triceps surae complex architecture in man: implications for muscle function. J Physiol. 1998;512:603-14. 
11. Curtis C, Stephens D, Clarke HM, Andrews D. The active movement scale: an evaluative tool for infants with obstetrical brachial plexus palsy. J Hand Surg. 2002;27:470-8,

12. Basheer H, Zelic V, Rabia F. Functional scoring system for obstetric brachial plexus palsy. J Hand Surg. 2000;25B:41-5.

13. Binzoni T, Bianchi S, Hanquinet S, Kaelin A, Sayegh Y, Dumont M, et al. Human gastronemius medialis pennation angle as a function of age, from new born to the elderly. J Physiol Anthropol Appl Hum Sci. 2001;20:293-8.

14. Lori S, Lolli F, Moletsi E, Bastianelli M, Gabbanini S, Saia V, et al. Muscleultrasound evaluation in healthy pediatric subjects: age related normative data. Muscle Nerve. 2018:58:245-50

15. Kawakami Y, Ichinose $Y$, Kubo K, Ito M, Fukunaga T. Architecture of contracting human muscles and it's functional significance. J Appl Biomech. 2000;16:88-98

16. Aagaard $P$, Andersen $\mathrm{L}$, Dyhre-Poulsen $P$, Leffers AM, Wanger A, Magnusson SP, et al. A mechanism for increased contractile strength of human pennate muscle in response to strength training: changes in muscle architecture. J Physiol. 2001;534:613-23.

17. Ikegawa S, Funato K, Tsunoda N, Kanehisa H, Fukunaga T, Kawakami Y. Muscle force per cross-sectional area is inversely related with pennation angle in strength trained athletes. J Strength Cond Res. 2008;22:128-31.

18. Fukunaga T, Miyatani M, Tachi M, Kouzaki M, Kawakami Y, Kanehisa H. Muscle volume is a major determinant of joint torque in humans. Acta Physiol Scand. 2001;172:249-55.

19. Strasser EM, Draskovits T, Praschak M, Quittan M, Graf A. Association between ultrasound measurements of muscle thickness, pennation angle, echogenicity and skeletal muscle strength in the elderly. Age. 2013;35:237788

20. Ali MS, Abdelaziem FH, Ali AA. Correlation between age, muscle thickness, spasticity, and functional ability in children with spastic hemiplegic cerebral palsy. Phys Ther Rehabil. 2019;6:6-8.

21. Morse Cl, Thom JM, Birch KM, Narici MV. Changes in triceps surae muscle architecture with sarcopenia. Acta Physiol Scand. 2005;183:291-8.

22. Reeves NJ, Maganaris CN, Ferretti G, Narici MV. Influence of stimulated microgravity on human skeletal muscle architecture and function. J Gravit Physiol. 2002;9:153-4.

23. Maarten D, Olivier R, Pietro E, lgor B, Narici M. Effect of 5 weeks horizontal bed rest on human muscle thickness and architecture of weight bearing and non-weight bearing muscles. J Appl Physiol. 2008:104:401-7.

24. Fukunaga $T$, Ichinose $Y$, Ito M, Kawakami Y, Fukashiro S. Determination of fascicle length and pennation in a contracting human muscle in vivo. $J$ Appl Physiol. 1997;82:354-8.

25. Nagano Y, Higashihara A, Edama M. Change in muscle thickness under contracting conditions following return to sports after a hamstring strain injury- a pilot study. AP-SMART. 2015;2:63-7.

\section{Publisher's Note}

Springer Nature remains neutral with regard to jurisdictional claims in published maps and institutional affiliations.

\section{Submit your manuscript to a SpringerOpen ${ }^{\circ}$ journal and benefit from:}

- Convenient online submission

- Rigorous peer review

- Open access: articles freely available online

- High visibility within the field

- Retaining the copyright to your article

Submit your next manuscript at $\boldsymbol{\nabla}$ springeropen.com 